\title{
Metacarpophalangeal Joint 1
}

National Cancer Institute

\section{Source}

National Cancer Institute. Metacarpophalangeal Joint 1. NCI Thesaurus. Code C102316.

A condyloid synovial joint within the first digit of the hand connecting the metacarpal to the proximal phalanx. 